\title{
Relationship between lenticular power and refractive error in children with hyperopia
}

This article was published in the following Dove Press journal:

Clinical Ophthalmology

22 March 2013

Number of times this article has been viewed

\section{Takeshi Tomomatsu \\ Shinjiro Kono \\ Shogo Arimura \\ Yoko Tomomatsu \\ Takehiro Matsumura \\ Yuji Takihara \\ Masaru Inatani \\ Yoshihiro Takamura}

Department of Ophthalmology, Faculty of Medical Sciences, University of Fukui, Fukuiken, Japan

Correspondence: Yoshihiro Takamura Department of Ophthalmology, Faculty of Medical Sciences,

University of Fukui, Eiheiji-cho,

Yoshida-gun, Fukui-ken,

910-I193, Japan

Tel $+8 \mid 776$ 6I 8I3।

Fax +81776618403

Email ytakamura@hotmail.com
Objectives: To evaluate the contribution of axial length, and lenticular and corneal power to the spherical equivalent refractive error in children with hyperopia between 3 and 13 years of age, using noncontact optical biometry.

Methods: There were 62 children between 3 and 13 years of age with hyperopia (+2 diopters [D] or more) who underwent automated refraction measurement with cycloplegia, to measure spherical equivalent refractive error and corneal power. Axial length was measured using an optic biometer that does not require contact with the cornea. The refractive power of the lens was calculated using the Sanders-Retzlaff-Kraff formula. Single regression analysis was used to evaluate the correlation among the optical parameters.

Results: There was a significant positive correlation between age and axial length $(P=0.0014)$; however, the degree of hyperopia did not decrease with aging $(P=0.59)$. There was a significant negative correlation between age and the refractive power of the lens $(P=0.0001)$ but not that of the cornea $(P=0.43)$. A significant negative correlation was observed between the degree of hyperopia and lenticular power $(P<0.0001)$.

Conclusion: Although this study is small scale and cross sectional, the analysis, using noncontact biometry, showed that lenticular power was negatively correlated with refractive error and age, indicating that lower lens power may contribute to the degree of hyperopia.

Keywords: emmetropization, axial length

\section{Introduction}

It is recognized that hyperopia in infants is associated with the pathogenesis of accommodative esotropia and amblyopia, but these conditions are preventable with the use of spectacles to correct refractive error. Atkinson et $\mathrm{al}^{1}$ reported that infants who wore spectacles had better visual acuity and a lower incidence of strabismus compared with infants with uncorrected hyperopia, at 4 years of age. Hyperopia gradually develops into emmetropia throughout infancy, especially in the first years of life..$^{2-5}$ The process of emmetropization can be considered the convergence of refractions toward a low hyperopic value, since it is reported that the average reduction in refractive error is a linear function of the initial level. ${ }^{6}$ However, there is substantial variability in the extent of emmetropization in infants with hyperopia. It is reported that the most hyperopic infants, those in excess of +5 diopters (D), tend not to emmetropize effectively. ${ }^{7}$ Thus, the mechanisms of emmetropization remain unclear.

The decline in hyperopia appears to be associated with increasing axial length. The cornea and lens could also be important contributors to emmetropization. The increase in axial length alone would result in an excessive shift towards myopia; however, this 
is offset by simultaneous changes in the lens that reduce power during infancy. ${ }^{6}$ If the power of the lens and cornea were low, even if the axial length increased, very hyperopic infants would not move rapidly toward emmetropia. Although recent longitudinal studies demonstrated that the refractive power of the lens decreases with age, the role of lens power in hyperopes is still unclear. ${ }^{8}$

Until now, amplitude modulation ultrasonography (A-scan) has been a conventional tool for measuring axial length and the corneal and lenticular power in infants. However, infants tend to fear the approach of the probe. The OA-1000 optical biometer (Tomey Corp, Nagoya, Japan) incorporates innovative technology to achieve high speed scanning and measurements in the depth direction (10 values in about 5 seconds) and sufficiently long scanning distance from the cornea to the retina with the interferometer. Unlike ultrasound biometry, this technique has the significant advantage of being quick and not requiring topical anesthesia for corneal applanation. Previous studies have reported that conventional ultrasound biometry resulted in a $54 \%$ measurement error in axial length. ${ }^{9,10}$ This noncontact technique appears to be valuable for the measurement of axial length, and corneal and lenticular power, in infants. In this study, we utilized the OA-1000 in a cross-sectional study evaluating the contribution of major ocular components, such as axial length, and corneal and lenticular power, to the degree of hyperopia of infants.

\section{Patients and methods}

Children who attended a pediatric ophthalmology clinic at the Fukui University Hospital between April 2010 and May 2012 and who received a diagnosis of hyperopia ( $+2 \mathrm{D}$ or more) without accommodative esotropia were enrolled into this cross-sectional study. In children with bilateral hyperopia, the eye with the higher degree of hyperopia was included in this study. This study was approved by the University of Fukui Institutional Review Board. Parents provided written informed consent according to the tenets of the Declaration of Helsinki, after all procedures were explained. Children with esotropia, other ophthalmological diseases, and history of ophthalmological surgery were excluded. Other exclusion criteria included previous strabismus surgery, albinism, nystagmus, and ocular or neurologic conditions. Cycloplegic refractive errors were determined 45 minutes after instillation (twice within 5-minute intervals) of cyclopentolate 1\% eyedrops. Refraction measurements were obtained using an auto ref/keratometer (TONOREF ${ }^{\mathrm{TM}}$ II; NIDEK, Gamagori, Japan). All patients were prescribed spectacles soon after hyperopia-related amblyopia was first diagnosed, and the full cylinder was always given.

Refractive errors were reported in terms of spherical equivalents. Corneal refraction (expressed in D) was also obtained from the auto ref/keratometer readings. Axial length measurements were performed by the same operator, using A-scan biometry (the Tomey OA-1000 optical biometer). The refractive power of the lens was calculated using the SandersRetzlaff-Kraff formula: $\mathrm{P}=\mathrm{A}-0.9 \mathrm{~K}-1.5 \mathrm{R}-2.5 \mathrm{~L}$, where $\mathrm{P}$ represents the intraocular lens power for emmetropia, $\mathrm{K}$ represents corneal refractive power (K-reading), $\mathrm{L}$ is the axial length, $\mathrm{A}$ is the $\mathrm{A}$-constant, and $\mathrm{R}$ represents the spherical equivalent refractive error. The same individual performed all measurements, using the same instruments. Univariate regression analysis was used to evaluate the correlation among the parameters. A $P$-value of 0.05 was accepted as statistically significant.

\section{Results}

There were 62 eyes, in 62 children with hyperopia (+2 D or more), included in the study. These children, 29 boys and 33 girls, were aged $7.14 \pm 1.99$ years (mean \pm standard deviation) at the time of data collection. The mean spherical equivalent refractive power was $+3.94 \pm 1.39 \mathrm{D}$, and the average degree of astigmatism was $+3.0 \pm 0.52 \mathrm{D}$. The mean visual acuity was $20 / 25$, and the axial length was $21.38 \pm 0.98 \mathrm{~mm}$. The mean refractive power of the lens and cornea were $+17.97 \pm 1.44 \mathrm{D}$ and $+43.30 \pm 1.77 \mathrm{D}$, respectively.

Our initial analysis found a significant negative correlation between spherical equivalent refractive power and axial length $(P<0.0001)$ (Figure 1$)$. To determine whether axial length increases with aging, we examined the relationship between age and axial length (Figure 2). A significant positive correlation was found between age and axial length $(P=0.0014)$. Since these results raise the possibility that increases in axial length with aging may lead to the relief of hyperopia, we assessed the relationship between age and spherical equivalent refractive power (Figure 3). Hyperopia tended to decrease with aging, but we found no significant correlation $(P=0.59)$. Factors related to the degree of refraction, other than axial length, include refractive power of the lens or cornea. Thus we investigated the relationship between age and lenticular refractive power (Figure 4). A significant negative correlation was observed between age and lenticular refractive power $(P=0.0001)$. Since decreases in the refractive power of the lens with aging may result in the progression of hyperopia, we examined the relationship 


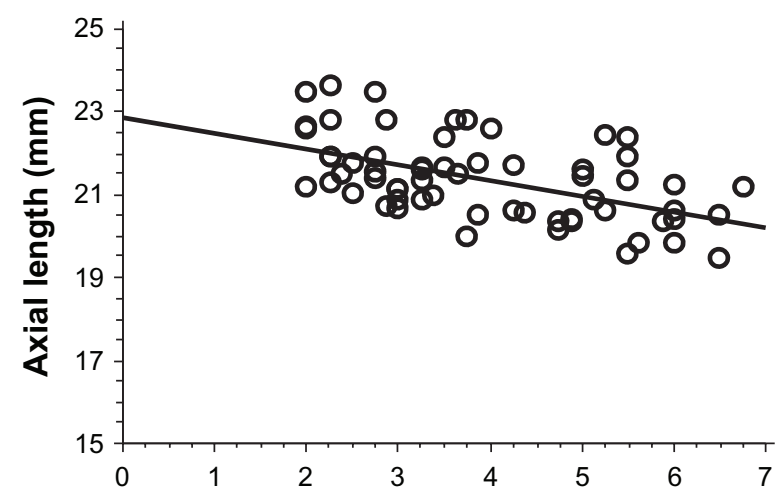

Spherical equivalent refractive power (D)

Figure I Relationship between spherical equivalent refractive power and axial length. Note: There was a significant negative correlation $\left(P<0.0001, R^{2}=0.29\right)$. Abbreviation: D, diopter.

between spherical equivalent refractive power and lenticular refractive power (Figure 5) and found a strong negative correlation $(P<0.0001)$.

We also investigated the relationship between the refractive power of the cornea and age, and a statistically significant correlation was not seen $(P=0.43)$. Also, there was no correlation between spherical equivalent refractive power and the refractive power of the cornea $(P=0.77)$ (Figure 6).

\section{Discussion}

Short axial length is an important determinant of the degree of hyperopia in children. Our data, obtained using noncontact optical biometry, showed a significant positive correlation between axial length and age. Also, a significant negative correlation between the lenticular power and age was observed. These data may imply the growth of axial length and the decreased lens power with aging, demonstrated by previous

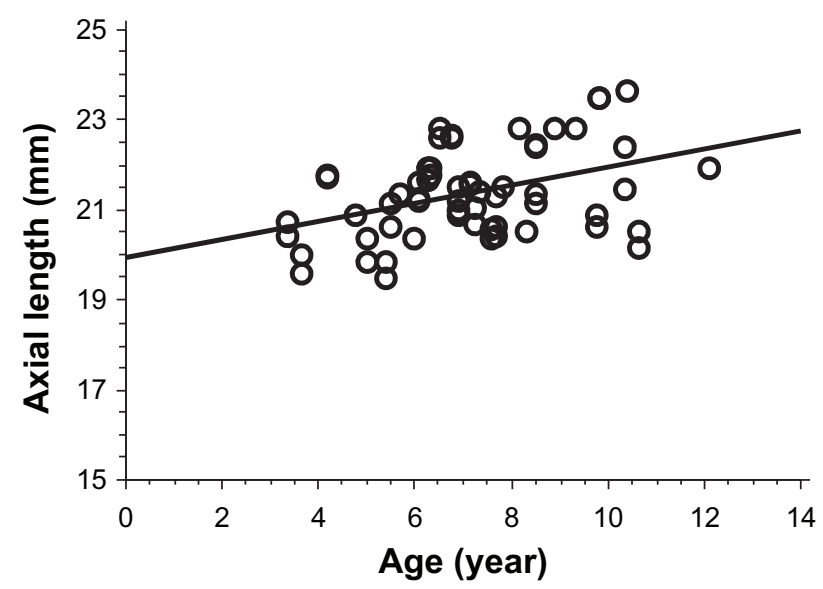

Figure 2 Correlation between age and axial length. Note: There was a significant positive correlation $\left(P=0.0014, R^{2}=0.16\right)$.

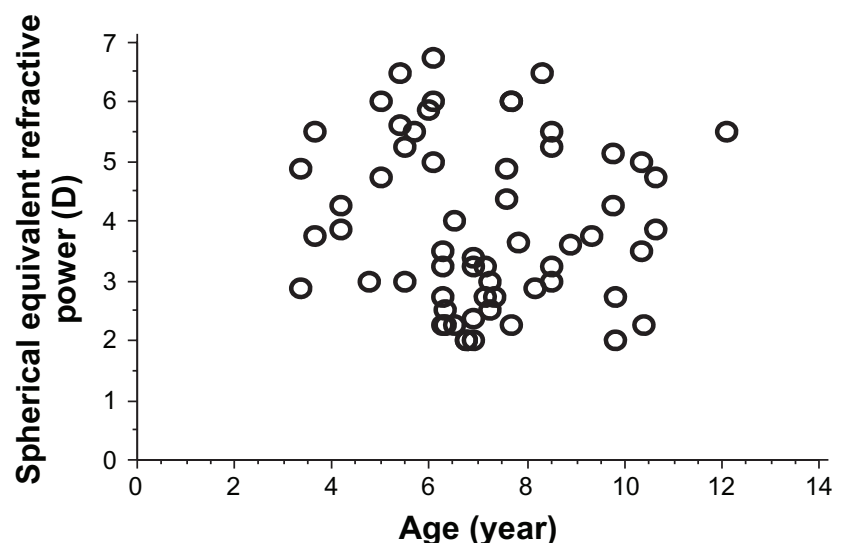

Figure 3 Correlation between age and spherical equivalent refractive power. Note: The relationship was insignificant $(P=0.59)$.

Abbreviation: D, diopter.

longitudinal studies. ${ }^{8,11-13}$ Moreover, we found that there was a significant negative correlation between lenticular power and the degree of hyperopia. In cases of severe hyperopia, the extensive growth of axial length and the limited decline of lens power are required to achieve emmetropization; however, our data suggested that the lens power is lower in eyes with a high degree of hyperopia. Therefore, weak lens power, as well as the limited growth of the axial length, may contribute to the high degree of hyperopia.

In this study, we used the optical biometer OA-1000, which provides noncontact and noninvasive measurements of axial length, based on the principle of low coherence interferometry. The optical biometry technique has higher precision and resolution than conventional ultrasound biometry. Rajan et al ${ }^{14}$ showed that the use of optical biometry to measure intraocular lens power improved refraction after cataract surgery by $16 \%$. The measurement using ultrasound biometry resulted in a mean shortening of the

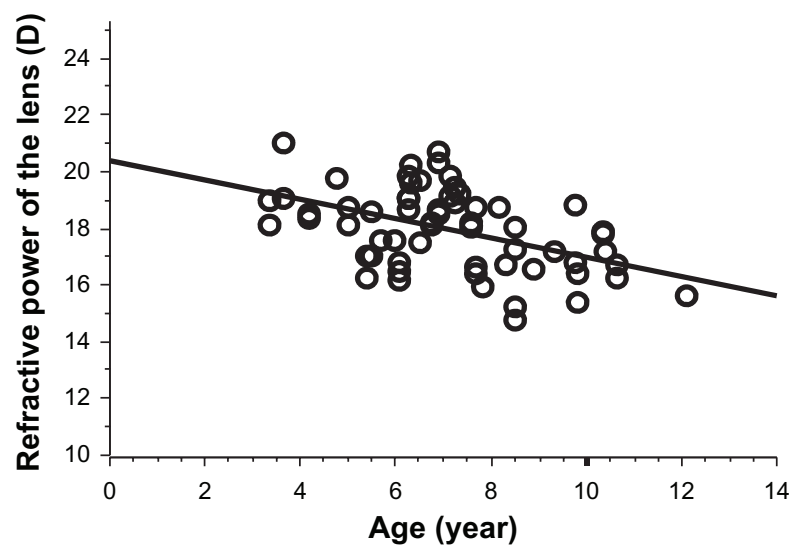

Figure 4 Correlation between age and refractive power of the lens. Note: There was a significant negative correlation $\left(P=0.000 \mathrm{I}, \mathrm{R}^{2}=0.22\right)$. Abbreviation: D, diopter. 


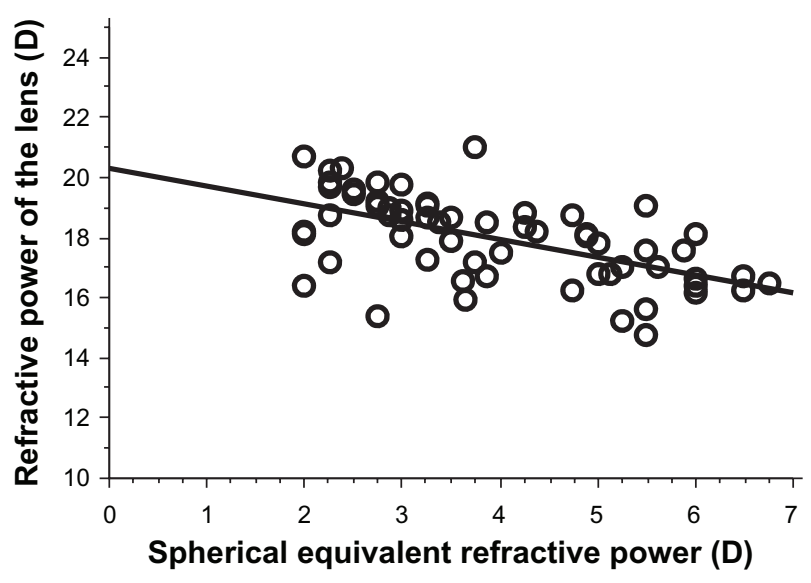

Figure 5 Correlation between spherical equivalent refractive power and lenticular power.

Note: There was a significant negative correlation $\left(P<0.000 \mathrm{I}, \mathrm{R}^{2}=0.33\right)$.

Abbreviation: D, diopter.

eye length. This error in measurement is thought to be due to the compression of the surface of the cornea by the probe. ${ }^{15}$ Moreover, measurements can be done without contact with the cornea, minimizing the risk of infection and increasing the effectiveness in those children who fear the approach of the probe and become uncooperative. Therefore the use of the optical biometry is considered valuable for estimating axial length in children.

We focused on the children who had hyperopia and set a spherical equivalent refraction cutoff. A lack of decline in the refractive error with aging could be due to the spherical equivalent refraction cutoff, since the emmetropizing children, in whom there was a reduction in hyperopia, might drop out of the sample of children at older ages. Actually, a significant correlation between age and refractive error was not found in our study.
This result may be also explained by the decrease in lenticular power with aging, leading to a shift toward hyperopia. Mutti et $\mathrm{al}^{11}$ showed that an increase in axial length leads to a decrease in refractive power (shift to myopia) and lens power (shift to hyperopia), in infants aged 3 and 9 months, and this correlation between increased axial length and decreased optical power was proposed as the "give and take" involved in emmetropization. Our data on children between 3 and 13 years of age showed a significant negative correlation between lenticular power and age. Consequently, it is possible that the myopic shift caused by increases in axial length is partially compensated by increased hyperopia that is caused by a reduction in lenticular power. This issue has been extensively studied using the Collaborative Longitudinal Evaluation of Ethnicity and Refractive Error (CLEERE) ${ }^{16}$ and the Sharable Content Object Reference Model (SCORM) data. ${ }^{8}$ In the SCORM study, 1747 children were classified into five groups: persistent hyperopia, emmetropizing hyperopia, persistent emmetropia, newly developed myopia, or persistent myopia. The lens power similarly decreased with aging in all groups except for the newly developed myopes, who showed significantly greater decrease in lens power. Therefore, it is likely that the decline of spherical equivalent refraction is not a specific physiological change in eyes with hyperopia. Axial elongation may remain the major biometric cause of myopia and emmetropization. Nevertheless, the loss of lens power needs to be assessed in more detail, since the regulation of the change in the lens power in relation to axial elongation or refractive change is still unclear. Further analysis of the lens power, calculated with the measurements of axial length using noncontact optical biometry, would be valuable for a more accurate investigation in a larger and longitudinal study.
A

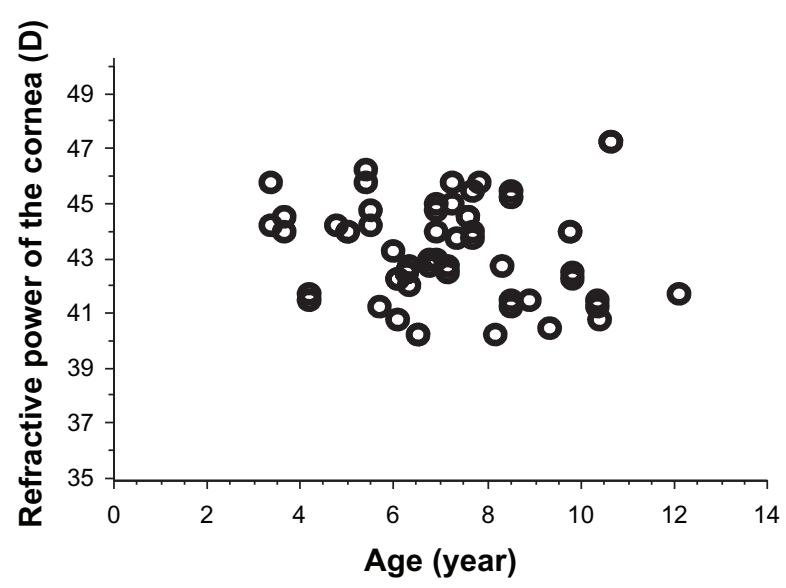

B

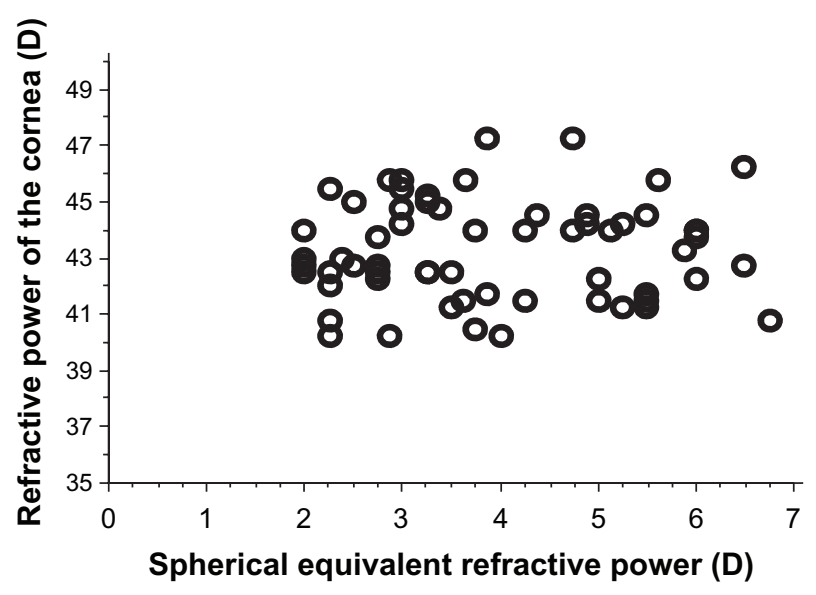

Figure 6 Correlation between refractive power of the cornea and $(\mathbf{A})$ age; and $(\mathbf{B})$ spherical equivalent refractive power.

Notes: The correlations were insignificant for both $(\mathbf{A})(P=0.43)$ and $(\mathbf{B})(P=0.77)$.

Abbreviation: D, diopter. 
Mechanisms involved in the decrease of lenticular power may involve, as observed by Zadnik et al, an actual lenticular thinning during childhood. ${ }^{12}$ During the period of rapid ocular growth, a significant association between increases in the equatorial diameter of the ocular globe and that of the lens has been found. ${ }^{6,13}$ As this change generates a force that stretches the lens in the equatorial plane and flattens the lens during ocular growth, the dioptric power of the lens decreases rapidly in childhood. ${ }^{6,13}$ On the other hand, Iribarren et $\mathrm{al}^{8}$ found a positive correlation between lens power and lens thickness and showed that there is a rapid loss of lens power and thickness up to the age of 10 years, with the lens slowly thickening after that age but continuing to lose power very slowly. The hyperopic shift in childhood, when the lens is increasing in thickness and curvature, is known as the lens paradox. This paradox would be resolved if increasing steepness of the gradient of refractive index leads to loss of lens power. ${ }^{6,17,18}$ It is possible that the compaction of the prenatal lens fibers (which results in lens thinning and flattening of lens surface) during childhood steepens the gradient of refractive index, making the lens lose internal power. ${ }^{8,19}$

In contrast to lenticular power, corneal power was not significantly correlated with age, consistent with previous reports that corneal power does not change between 6 and 9 years of age. ${ }^{6,16}$ Sorsby et a ${ }^{20}$ showed that corneal power declines only minimally, from approximately $+43.0 \mathrm{D}$ at the age of 3 years to $+42.7 \mathrm{D}$ at the age of 14 years. Taken together, this suggests that corneal power has little influence on spherical equivalent refractive errors in childhood.

The clarity of visual input is considered to be the major contributing factor to the control of eye growth and emmetropization. ${ }^{6,21}$ Several studies have provided evidence that full correction of the hyperopic refractive error with eyeglasses may hinder emmetropization. ${ }^{4,22-24}$ According to these studies, eliminating the retinal blur with appropriate lenses could remove the stimulus for the myopic shift toward emmetropia in hyperopic children. In contrast, Demirkilinç et $\mathrm{al}^{7}$ suggested that partial or full correction of hyperopia has similar effects on refractive development as in accommodative esotropia. Thus, it is possible that the way of correction for hyperopia may influence on the refractive development. In this study we excluded patients with partial optical correction of hyperopia.

In this study, we analyzed axial length and lens power in children with hyperopia, using noncontact biometry, and showed a significant negative correlation between lens power and refractive error in hyperopes, indicating that lower lens power may contribute to severe hyperopia. However, further longitudinal study is necessary to clarify whether severe hyperopes with lower lens power are slowly driven in an emmetropic direction.

\section{Disclosure}

The authors report no conflicts of interest in this work.

\section{References}

1. Atkinson J, Braddick O, Robier B, et al. Two infant vision screening programmes: prediction and prevention of strabismus and amblyopia from photo- and videorefractive screening. Eye (Lond). 1996;10(Pt 2):189-198.

2. Atkinson J, Anker S, Bobier W, et al. Normal emmetropization in infants with spectacle correction for hyperopia. Invest Ophthalmol Vis Sci. 2000;41(12):3726-3731.

3. Yahalom C, Tzur V, Blumenfeld A, et al. Refractive profile in oculocutaneous albinism and its correlation with final visual outcome. $\mathrm{Br} J$ Ophthalmol. 2012;96(4):537-539.

4. Ingram RM, Arnold PE, Dally S, Lucas J. Emmetropisation, squint, and reduced visual acuity after treatment. Br J Ophthalmol. 1991; 75(7):414-416.

5. Ehrlich DL, Braddick OJ, Atkinson J, et al. Infant emmetropization: longitudinal changes in refraction components from nine to twenty months of age. Optom Vis Sci. 1997;74(10):822-843.

6. Brown NP, Koretz JF, Bron AJ. The development and maintenance of emmetropia. Eye (Lond). 1999;13(Pt 1):83-92.

7. Demirkilinç Biler E, Uretmen O, Köse S. The effect of optical correction on refractive development in children with accommodative esotropia. J AAPOS. 2010;14(4):305-310.

8. Iribarren R, Morgan IG, Chan YH, Lin X, Saw SM. Changes in lens power in Singapore Chinese children during refractive development. Invest Ophthalmol Vis Sci. 2012;53(9):5124-5130.

9. Norrby S. Sources of error in intraocular lens power calculation. J Cataract Refract Surg. 2008;34(3):368-376.

10. Olsen T. Sources of error in intraocular lens power calculation. $J$ Cataract Refract Surg. 1992;18(2):125-129.

11. Mutti DO, Mitchell GL, Jones LA, et al. Axial growth and changes in lenticular and corneal power during emmetropization in infants. Invest Ophthalmol Vis Sci. 2005;46(9):3074-3080.

12. Zadnik K, Mutti DO, Fusaro RE, Adams AJ. Longitudinal evidence of crystalline lens thinning in children. Invest Ophthalmol Vis Sci. 1995;36(8):1581-1587.

13. Mutti DO, Zadnik K, Fusaro RE, Friedman NE, Sholtz RI, Adams AJ. Optical and structural development of the crystalline lens in childhood. Invest Ophthalmol Vis Sci. 1998;39(1):120-133.

14. Rajan MS, Keilhorn I, Bell JA. Partial coherence laser interferometry vs conventional ultrasound biometry in intraocular lens power calculations. Eye (Lond). 2002;16(5):552-556.

15. Findl O, Drexler W, Menapace R, Heinzl H, Hitzenberger CK, Fercher AF. Improved prediction of intraocular lens power using partial coherence interferometry. J Cataract Refract Surg. 2001;27(6):861-867.

16. Zadnik K, Manny RE, Yu JA, et al; Collaborative Longitudinal Evaluation of Ethnicity and Refractive Error (CLEERE) Study Group. Ocular component data in schoolchildren as a function of age and gender. Optom Vis Sci. 2003;80(3):226-236.

17. Dubbelman M, Van der Heijde GL. The shape of the aging human lens: curvature, equivalent refractive index and the lens paradox. Vision Res. 2001;41(14):1867-1877.

18. Hashemi H, Iribarren R, Morgan IG, Khabazkhoob M, Mohammad K, Fotouhi A. Increased hyperopia with ageing based on cycloplegic refractions in adults: the Tehran Eye Study. Br J Ophthalmol. 2010; 94(1):20-23.

19. Augusteyn RC. On the growth and internal structure of the human lens. Exp Eye Res. 2010;90(6):643-654. 
20. Sorsby A, Benjamin B, Sheridan M, Stone J, Leary GA. Refraction and its components during the growth of the eye from the age of three. Memo Med Res Counc. 1961;301(Special):1-67.

21. Flitcroft DI. A model of the contribution of oculomotor and optical factors to emmetropization and myopia. Vision Res. 1998;38(19): 2869-2879.

22. Baker JD, Parks MM. Early-onset accommodative esotropia. Am J Ophthalmol. 1980;90(1):11-18.
23. Friedburg D, Klöppel KP. [Early correction of hyperopia and astigmatism in children leads to better development of visual acuity.] Klin Monbl Augenheilkd. 1996;209(1):21-24. [German.]

24. Troilo D. Neonatal eye growth and emmetropisation-a literature review. Eye (Lond). 1992;6(Pt 2):154-160.
Clinical Ophthalmology

\section{Publish your work in this journal}

Clinical Ophthalmology is an international, peer-reviewed journal covering all subspecialties within ophthalmology. Key topics include: Optometry; Visual science; Pharmacology and drug therapy in eye diseases; Basic Sciences; Primary and Secondary eye care; Patient Safety and Quality of Care Improvements. This journal is indexed on

\section{Dovepress}

PubMed Central and CAS, and is the official journal of The Society of Clinical Ophthalmology (SCO). The manuscript management system is completely online and includes a very quick and fair peer-review system, which is all easy to use. Visit http://www.dovepress.com/ testimonials.php to read real quotes from published authors. 